\title{
Clinical Features of Bell's Palsy in Children and Outcomes of Physical Therapy: A Retrospective Study
}

\author{
Duygu Cubukcu, ${ }^{1}$ Unsal Yilmaz, ${ }^{2}$ Hakan Alkan, ${ }^{3}$ Ferda Metinkisi, ${ }^{1}$ and Muazzez Ozcan ${ }^{4}$ \\ ${ }^{1}$ Department of Physical Medicine and Rehabilitation, Dr. Behcet Uz Children's Diseases Training and Research Hospital, \\ Sezer Dogan Street No. 11, Konak, 35210 Izmir, Turkey \\ ${ }^{2}$ Department of Pediatric Neurology, Dr. Behcet Uz Children's Diseases Training and Research Hospital, Sezer Dogan Street No. 11, \\ Konak, 35210 Izmir, Turkey \\ ${ }^{3}$ Department of Physical Medicine and Rehabilitation, Pamukkale University Faculty of Medicine, Kınılı 20070, Denizli, Turkey \\ ${ }^{4}$ Department of Otorhinolaryngology, Dr. Behcet Uz Children's Diseases Training and Research Hospital, Sezer Dogan Street No. 11, \\ Konak, 35210 Izmir, Turkey
}

Correspondence should be addressed to Hakan Alkan; alkangsc@yahoo.com

Received 10 October 2013; Accepted 9 November 2013

Academic Editors: S. Eyigor and E. E. Hansson

Copyright (C) 2013 Duygu Cubukcu et al. This is an open access article distributed under the Creative Commons Attribution License, which permits unrestricted use, distribution, and reproduction in any medium, provided the original work is properly cited.

\begin{abstract}
The aim of this study was to describe clinical features of Bell's palsy (BP) in children and outcomes of physical therapy interventions. Medical records of 186 patients with diagnosis of BP were reviewed. The severity of facial nerve dysfunction was graded according to House Brackmann Facial Nerve Grading Scale (HB FGS). After one month, patients who had HB FGS grade three or more were included in a physical therapy programme consisting of superficial heat, massage, exercises, and electrical stimulation. Among 186 children with BP, 167 children had a severity of HB FGS grade three or more and 19 had the incomplete type at the time of the diagnosis. After one month follow-up, 144 patients fully recovered, 35 patients recovered partially, while 7 patients had no recovery. Patients who had not fully recovered one month after onset of paralysis were included in the physical therapy programme. There was statistically significant difference between pretreatment and posttreatment HB FGS scores in patients who had not fully recovered one month after onset of paralysis. We concluded that the prognosis of BP in children seems to be good with higher recovery rate and physical therapy programme might be an effective method to facilitate recovery in patients with poor prognosis.
\end{abstract}

\section{Introduction}

The most common type of facial paralysis in children is Bell's palsy (BP), which is defined as an acute, idiopathic, unilateral paralysis of the facial nerve without any associated disorders. Known congenital, genetic, and acquired causes of facial nerve paralysis need to be ruled out before the diagnosis of $\mathrm{BP}$ is made [1]. It has an incidence rate of 18.8 cases per 100.000 in children younger than 18 years old [2]. BP is an important cause of disability in some pediatric patients with major facial dysfunction and reduced quality of life [3].

Facial functions are multidimensional, serving emotional, social, and physical aspects of a person's health.
Incomplete recovery from facial nerve damage has significant social and psychological consequences. The prognosis of BP in pediatric population is generally good with a high rate of recovery. In spite of high complete recovery rate, some patients with BP do not achieve even satisfactory recovery. Moreover children with complete facial palsy and absence of recovery within 3 weeks may suffer poor outcomes as frequently as adults $[4,5]$. In contrast with adults, the natural course of BP in children is not well documented and there are still many unresolved issues regarding the treatment and prognosis. Even though the treatment and prognosis of BP differ among children and adults, there are only a few studies focused on children [6-10]. Furthermore to our 
knowledge, there is no study in the literature which evaluates the outcomes of physical therapy interventions solely in children with BP.

Knowledge on the course of disease and clinical features of BP in children largely depends on the studies of BP among adults. Little is known regarding the clinical features and outcomes of physical therapy programme among children with BP. In this study, we aimed to investigate clinical features of $\mathrm{BP}$ as well as the outcomes of physical therapy programme in pediatric patients who had not fully recovered one month after onset of paralysis.

\section{Patients and Methods}

Medical records of patients younger than 18 years old, attending one of the Physical Medicine and Rehabilitation outpatient clinics at Dr. Behçet Uz Children's Diseases Training and Research Hospital between January 2007 and December 2012, were retrospectively reviewed and children who had the diagnosis of BP were identified. Children with facial paralysis due to central causes were excluded. The study protocol was approved by local ethics committee.

Each child had been examined by a pediatric neurologist, an otolaryngologist, an ophthalmologist, and a physiatrist during initial evaluation as it is a routine in Dr. Behçet Uz Children's Diseases Training and Research Hospital. All patients were referred to Physical Medicine and Rehabilitation outpatient clinic by pediatric neurologist. Each child was assessed by pediatric neurologist as well as a physiatrist with a standardized work-up and follow-up. Following a thorough history, a detailed physical and neurologic examination were performed. Moreover, selected laboratory testing such as serological viral markers for various viruses, including human immunodeficiency, Epstein-Barr, hepatitis B, and herpes simplex virus and also serologic test to rule out Lyme disease were obtained based on the suspected diagnosis. All patients in whom secondary facial nerve palsy is suspected a diagnostic work-up for the presence or absence of possible causes had been promptly initiated.

Detailed information including demographic characteristics, time of presentation in regard to the season of the year, medical history, family history and outcome were collected. The diagnosis of BP had been made when no obvious cause was identified by medical history, physical examination, or radiologic studies.

The severity of facial nerve dysfunction and the recovery rate after treatment were evaluated according to HouseBrackmann Facial Nerve Grading Scale (HB FGS), an easily administered, widely used clinical system for grading recovery from facial nerve paralysis caused by damaged to lower motor neurons [11]. On this scale, grade I represents normal face function while grade VI refers to total paralysis; II mean slight weakness (mild dysfunction); III moderate dysfunction; IV moderate to severe; V represents severe dysfunction. In IV and V grades patient can not close the eye and could only barely move the mouth at V [11].

In patients with BP, when HB FGS was grade three or more, oral prednisolone therapy was initiated with a dose of $2 \mathrm{mg} / \mathrm{kg}$ up to $60 \mathrm{mg}$ daily for 5 days followed by 5 days taper. When a viral infection is suspected, antiviral medication was initiated. In addition, a home based exercise programme consisting of training the facial muscles following a standardized protocol were initiated in all patients at the time of the diagnosis. A standard exercise handout for home programmes was given to each subject on the initial consultation with the physiatrist. Exercises were chosen to include different muscle groups supplied by each branch of the facial nerve repeated 10 times a day. Exercises were performed for both sides of the face by using a mirror to promote symmetry and feedback. The exercises included in the home programme were shown to the patient on the initial treatment.

Recovery was defined as the achievement of HB FGS grade one or two. All children with BP were followed up until complete recovery. Recovery was assessed with followup visits once a week during the first month and subsequently every month until satisfactory recovery.

Patients who had HB FGS grade more than two within one month after onset of paralysis, were included in the physical therapy programme. The physical therapy programme consisted of infrared application, massage to the face musculature, electrical stimulation, and exercise. Local superficial heat therapy by the application of infrared was performed for $15 \mathrm{~min} / \mathrm{session}$ for the facial muscles prior to electrical stimulation (ES), massage or exercises. Massage was applied for 10 minutes to both sides of the face. ES was aplied to the facial muscles which have no active motion or muscle strength lower than three out of five. For stimulating muscles which is completely denervated, interrupted galvanic stimulation of $100 \mathrm{~ms}$ rectangular pulses may be given at rate of 1 pulse/s for 30-100 contraction/session. During each session, ES should be stopped once muscle fatigue occurs. ES was stopped as soon as the active motion of muscle seen. Exercises carried out on consultation with the physiotherapist were based on current trends of the facial retraining rehabilitation programme. This physical therapy programme was applied once daily for 5 days a week in patients who had HB FGS grade more than two. Median number of physical therapy sessions were 10 (range 5-32).

\section{Statistical Analysis}

All statistical analyses were performed using the SPSS version 17.0 for Windows software program (SPSS Inc., Chicago, IL, USA). Kolmogorov-Smirnov test was used to assess whether continuous variables were normally distributed or not. Descriptive statistics were used for the demographic characteristics. For continuous variables, the significance of the differences was analyzed using Student's $t$-test for normally distributed variables while categorical variables were analyzed with a chi-square test. Wilcoxon Singed Rank test was used to analyze the difference between the pretreatment and postreatment HB FGS. In all analyses, $P$ values $<0.05$ were considered statistically significant. 
TABLE 1: Demographic characteristics and clinical features in patients with Bell's palsy.

\begin{tabular}{|c|c|}
\hline & Patients $(n=186)$ \\
\hline \multicolumn{2}{|l|}{ Gender, $n(\%)$} \\
\hline Male & $79(42.5 \%)$ \\
\hline Female & $107(57.5 \%)$ \\
\hline Age (years) $($ mean $\pm \mathrm{SD})$ & $9.56 \pm 3.45$ \\
\hline \multicolumn{2}{|l|}{ Affected side, $n(\%)$} \\
\hline Right & $97(52.2 \%)$ \\
\hline Left & $89(47.8 \%)$ \\
\hline \multicolumn{2}{|l|}{ Season, $n(\%)$} \\
\hline Winter & $42(22.6 \%)$ \\
\hline Spring & $47(25.3 \%)$ \\
\hline Summer & $30(16.1 \%)$ \\
\hline Autumn & $67(36 \%)$ \\
\hline \multicolumn{2}{|l|}{ Severity, $n(\%)$} \\
\hline Mild & $19(10.2 \%)$ \\
\hline Severe & $167(89.8 \%)$ \\
\hline \multicolumn{2}{|l|}{ Recurrence, $n(\%)$} \\
\hline No & $174(93.5 \%)$ \\
\hline Yes & $12(6.5 \%)$ \\
\hline \multicolumn{2}{|l|}{ Family history, $n(\%)$} \\
\hline No & $177(95.2 \%)$ \\
\hline Yes & $9(4.8 \%)$ \\
\hline \multicolumn{2}{|l|}{ HB FGS, $n(\%)$} \\
\hline Grade II & $19(10.2 \%)$ \\
\hline Grade III & $31(16.7 \%)$ \\
\hline Grade IV & $44(23.7 \%)$ \\
\hline Grade V & $77(41.4 \%)$ \\
\hline Grade VI & $15(8.0 \%)$ \\
\hline \multicolumn{2}{|l|}{ After first month, $n(\%)$} \\
\hline Full recovery & $144(77.4 \%)$ \\
\hline Partial recovery & $35(18.8 \%)$ \\
\hline No recovery & $7(3.8 \%)$ \\
\hline \multicolumn{2}{|l|}{ After rehabilitation, $n(\%)$} \\
\hline Full recovery & $35(83.3 \%)$ \\
\hline Partial recovery & $7(16.7 \%)$ \\
\hline No recovery & - \\
\hline
\end{tabular}

HB FGS: House Brackmann Facial Nerve Grading scale.

\section{Results}

A total of 186 patients with BP reviewed of whom 79 were male and 107 were female, with a mean age of $9.56 \pm 3.45$ years ranging from 0 to 18 years. The majority of patients were seen in the autumn (67) and spring (47), respectively. The smallest number of the patients were seen on summer. There were 97 right and 89 left facial palsies. Out of 186 patients $9(4.8 \%)$ had a positive family history of BP and 12 (6.5\%) children had recurrent facial nerve palsy (Table 1 ).

Also, we could not demonstrate a significant difference on demographic and clinical features of BP patients with mild or severe disease $(P>0.05)$. On the other hand children
TABLE 2: Demographic and clinical features of Bell's palsy patients according to severity of disease.

\begin{tabular}{lccc}
\hline & $\begin{array}{c}\text { Mild } \\
(n=19)\end{array}$ & $\begin{array}{c}\text { Severe } \\
(n=167)\end{array}$ & $P$ value \\
\hline Gender, $n$ (\%) & $11(57.9 \%)$ & $68(40.7 \%)$ & 0.51 \\
$\quad$ Male & $8(42.1 \%)$ & $99(59.3 \%)$ & \\
$\quad$ Female & $8.84 \pm 2.69$ & $9.64 \pm 3.52$ & 0.21 \\
Age (years) \\
(mean \pm SD) & & & 0.22 \\
Season, $n(\%)$ & $1(5.3 \%)$ & $41(24.6 \%)$ & \\
$\quad$ Winter & $5(26.3 \%)$ & $42(25.1 \%)$ & \\
$\quad$ Spring & $5(26.3 \%)$ & $25(15.0 \%)$ & \\
$\quad$ Summer & $8(42.1 \%)$ & $59(35.3 \%)$ & \\
$\quad$ Autumn & & & \\
Affected side, $n(\%)$ & $9(47.4 \%)$ & $88(52.7 \%)$ & \\
$\quad$ Right & $10(52.6 \%)$ & $79(47.3 \%)$ & \\
$\quad$ Left & & & \\
Recurrence, $n(\%)$ & $19(100 \%)$ & $155(92.8 \%)$ & \\
No & $0(0 \%)$ & $12(7.2 \%)$ & \\
$\quad$ Yes & & & \\
After first month, $n(\%)$ & & & \\
Full recovery & $19(100 \%)$ & $104(62.3 \%)$ & \\
Partial recovery & $0(0 \%)$ & $56(33.5 \%)$ & \\
No recovery & $0(0 \%)$ & $7(4.2 \%)$ & \\
\hline
\end{tabular}

${ }^{*}$ Statistically significant difference.

with mild BP had significantly higher full recovery rate than children with severe BP $(P<0.05)$ (Table 2$)$.

Among 186 children with BP, 167 (89.8\%) children had a severity of HB FGS grade three or more and 19 (10.2\%) had the incomplete type at the time of the diagnosis. After one month follow-up, 144 (77.4\%) patients fully recovered, 35 (18.8\%) patients recovered partially while 7 patients $(3.8 \%)$ had no recovery.

A total of 42 patients who had not fully recovered one month after onset of paralysis, were included in the physical therapy programme. After the physical therapy programme at a mean 10 sessions totally; $35(83.3 \%)$ patients out of 42 were fully recovered while $7(16.7 \%)$ had partial recovery. There was a statistically significant difference between pretreatment and posttreatment HB FGS scores $(P<0.001)$ (Table 3$)$. Finally at the end of one year follow-up, all patients except three were fully recovered. Moreover sequela such as contracture and synkinesis were not shown in children with BP.

\section{Discussion}

In this study, we describe a retrospective analysis designed specifically to determine the clinical features of BP and outcomes of physical therapy programme in pediatric patients with BP. Our results demonstrated that the majority of patients were diagnosed in spring and also had a complete BP. However the prognosis of $\mathrm{BP}$ was good with higher recovery 
TABLE 3: Outcome of physical therapy programme in children with Bell's palsy who had no full recovery within one month after onset of paralysis.

\begin{tabular}{lccc}
\hline & $\begin{array}{c}\text { Before } \\
\text { rehabilitation } \\
(n=42)\end{array}$ & $\begin{array}{c}\text { After } \\
\text { rehabilitation } \\
(n=42)\end{array}$ & $P$ value \\
\hline $\begin{array}{l}\text { According to } \\
\text { HB FGS, } n \text { (\%) }\end{array}$ & & & $<0.001$ \\
$\quad$ Full recovery & $0(0 \%)$ & $35(83.3 \%)$ & \\
Partial recovery & $35(83.3 \%)$ & $7(16.7 \%)$ & \\
$\begin{array}{l}\text { No recovery } \\
\text { HB FGS }\end{array}$ & $7(16.7 \%)$ & $0(0 \%)$ & \\
$\begin{array}{l}\text { Median } \\
\text { (minimum- }\end{array}$ & $4(3-6)$ & $2(1-4)$ & $<0.001$ \\
maximum) & & & \\
\hline
\end{tabular}

HB FGS: House Brackmann Facial Nerve Grading scale.

rate. On the other hand physical therapy programme seems to be an effective method to facilitate recovery in patients with poor prognosis.

It is important that we have a clear understanding about the clinical features of BP. In a recent retrospective study which 51 cases $(60.7 \%)$ were classified as BP, it was demonstrated that the number of girls and boys were equal with 42 patients of each gender and the mean age was 9.6 years. Also the facial palsy affected the right side in 40 patients $(47.6 \%)$, the left side in 43 cases $(51.2 \%)$ and in one patient (1.2\%) it appeared bilaterally [6]. In another retrospective study it was reported that the male to female ratio was 0.9 in children with $\mathrm{BP}$ as well as the ratio of the affected side was left/right 1/0.8 also the mean age was 6 years 7 months and the peak age distribution was under 3 years [7]. The mean age in the pediatric population lies between 5 and 11 years with no significant difference between female and male patients $[7,12,13]$. Comparable to these studies, we found no difference concerning the gender or the affected side of the face.

Reports of children with recurrent BP is uncommon. Recurrent BP was found in only two children (4.3\%) out of 47 children with BP in a Thai study [9]. In another study, 3 (10.3\%) out of 29 children with BP admitted during 19952003 had recurrent facial nerve palsy [7]. We showed an incidence of $6.5 \%$ of 186 pediatric patients had recurrent BP, which was similar to these studies. On the other hand exposure to cold may trigger the BP. However, it is not clear that cold has a significant independent effect on risk of disease. Although seasonal variations in BP rates were observed, they were usually lower in summer. Cambell and Brundage [14] demonstrated that incidence rates for $\mathrm{BP}$ during colder months of the year were consistently higher than incidence rates during warmer months of the year.

Prognosis of BP in pediatric population were rarely studied. In a recent retrospective study it was reported that 75 patients $(89.3 \%)$ recovered completely among 84 children with peripheral facial palsy of whom 51 cases $(60.7 \%)$ were classified as BP [6]. In the Copenhagen facial nerve study which evaluated the natural history of 463 children with peripheral facial nerve palsy over a 25 -year period, full recovery was achieved by $90 \%$ of these children [15]. In another retrospective study, the recovery rate within 3 weeks for children with BP was $68.8 \%$ whereas the complete recovery rate was high, 31 episodes (96.9\%) had complete recovery within 32 episodes of BP in 29 children at the end of the 7 months from onset [7]. A recent study Jenke et al. reported a $97 \%$ spontaneous complete recovery of their patients, even though only $3.7 \%$ had been treated with corticosteroids [8]. In spite of high complete recovery rate, children with complete facial palsy and absence of recovery within 3 weeks can be indicators of poor prognosis in patients with BP (4). In accordance with these findings we also found that complete recovery was achieved by $77.4 \%$ children with $\mathrm{BP}$ and partial recovery was $18.8 \%$ while $3.8 \%$ achieved no recovery after the first month. We also demonstrated that out of remaining 42 patients who had not fully recovered after the first month 35 (83.3\%) patients got full recovery while 7 (16.7\%) had partial recovery after the physical therapy programme. Moreover, at the end of one year we observed that all except three patients had full recovery. In contrast to these, in a recent study [16] it was concluded that facial nerve palsy in children is not as benign as it was previously supposed. Surprisingly this study found that $21 \%$ of the participants had persistent mild to moderate dysfunction.

The prognosis of BP is primarily related to the severity of the initial dysfunction. We can say that clinically incomplete lesions tends to recover. Patients with incomplete facial nerve palsy have a better prognosis compared with those who have complete paralysis $[15,17]$. This is in accordance with results of our study which demonstrated children with mild BP had significantly higher full recovery rate than children with severe BP.

It is still controversial whether children with BP should be given any medical treatment or applied physical therapy programme. Main goals of treatment are to expedite recovery without any sequela such as contracture and synkinesis [4]. Evaluation of any therapy for BP is difficult due to the high rates of spontaneous and complete recovery. The main question is whether results would be better if some treatment were given [18]. Most studies include only adults or a mixed population. Although a systematic review showed a higher recovery rate with corticosteroid and antiviral treatments, these data mostly refer to adults and trials including children are sparse [19]. Unüvar et al. described a quicker recovery, but no better outcome of facial palsy in children when treated with corticosteroids [20]. Currently, there is no sufficient evidence showing any clear benefit in the use of steroids or antiviral agents in the treatment of acute peripheral facial palsy in children [6]. However, in the present study when a patient had HB FGS grade three or more, steroid was initiated with a dose of $2 \mathrm{mg} / \mathrm{kg}$ up to $60 \mathrm{mg}$ daily and whenever a viral infection is suspected antiviral medication was also initiated. Since all of the patients had received treatment, we could not make any conclusions on its effect on the natural outcome of the disease.

$\mathrm{BP}$ is commonly treated with various rehabilitation strategies and therapeutic modalities. There are many questions 
about their efficacy and effectiveness. Exercise is the most commonly used method for the rehabilitation of BP. Thermal methods, electrotherapy, massage and biofeedback are other forms that could be used for rehabilitation [18, 21]. The Cochrane Systematic Review [21] concludes that high-quality evidence to support significant benefit or harm from any physical therapy for idiopathic facial paralysis is lacking. There is, however, low-quality evidence that facial exercises can help improve facial function, mainly for people with moderate paralysis and chronic cases, and that facial exercise reduces sequelae in acute cases [21]. The possibility that facial exercise reduces time to recover and sequelae needs confirming with good quality randomised controlled trials. Physical therapy agents are often used in the treatment of idiopathic facial paralysis in adults, but the effectiveness is not known exactly. It is still unclear whether rehabilitation affects the natural course of BP. To our knowledge this is the first study that found physical therapy programme to be an effective method to facilitate recovery in children with $\mathrm{BP}$ who had not fully recovered within one month after onset of paralysis.

Major limitations of this study are its retrospective design rather than longitudinal follow up, relatively small number of patients who were included in the physical therapy programme, unequal number of physical therapy sessions and due to the lack of control group, difficulty in determining the outcome of treatment. Since this is a retrospective study, we were not able to assess the separate contribution of all possible confounders that have been associated with severity and also outcome of BP, which reduced the statistical power of our study. Despite these limitations, to our knowledge this is the first study focusing on the effect of comprehensive physical therapy programme in children with BP who had partial recovery after one month. Further longitudinal, prospective studies are warranted to evaluate the efficacy of physical therapy interventions in children with BP who had poor prognosis.

\section{Conclusions}

Prognosis of BP seems to be good with higher recovery rate in children with $\mathrm{BP}$ and physical therapy programme might be an effective method to facilitate recovery in patients who had not fully recovered within one month after onset of paralysis.

\section{Conflict of Interests}

The authors declare that there is no conflicts of interests.

\section{References}

[1] M. Lorch and S. J. Teach, "Facial nerve palsy: etiology and approach to diagnosis and treatment," Pediatric Emergency Care, vol. 26, no. 10, pp. 763-769, 2010.

[2] A. Rowhani-Rahbar, R. Baxter, B. Rasgon et al., "Epidemiologic and clinical features of Bell's palsy among children in Northern California," Neuroepidemiology, vol. 38, no. 4, pp. 252-258, 2012.
[3] J. Shargorodsky, H. W. Lin, and Q. Gopen, "Facial nerve palsy in the pediatric population," Clinical Pediatrics, vol. 49, no. 5, pp. 411-417, 2010.

[4] N. J. Holland and G. M. Weiner, "Recent developments in Bell's palsy," The British Medical Journal, vol. 329, no. 465, pp. 553-557, 2004.

[5] J. Holland, "Bell's palsy," Clinical Evidence, vol. 2008, article $1204,2008$.

[6] F. Drack and M. Weissert, "Outcome of peripheral facial palsy in children-a catamnestic study," European Journal of Paediatric Neurology, vol. 17, no. 2, pp. 185-191, 2013.

[7] W. X. Chen and V. Wong, "Prognosis of Bell's palsy in childrenanalysis of 29 cases," Brain and Development, vol. 27, no. 7, pp. 504-508, 2005.

[8] A. C. Jenke, L. M. Stoek, M. Zilbauer, S. Wirth, and P. Borusiak, "Facial palsy: etiology, outcome and management in children," European Journal of Paediatric Neurology, vol. 15, no. 3, pp. 209213, 2011.

[9] K. Dhiravibulya, “Outcome of Bell's palsy in children," Journal of the Medical Association of Thailand, vol. 85, pp. 334-339, 2002.

[10] C. H. Wang, Y. C. Chang, H. M. Shih, C. Y. Chen, and J. C. Chen, "Facial palsy in children: emergency department management and outcome," Pediatric Emergency Care, vol. 26, no. 2, pp. 121$125,2010$.

[11] J. W. House and D. E. Brackmann, "Facial nerve grading system," Otolaryngology-Head and Neck Surgery, vol. 93, no. 2, pp. 146-147, 1985.

[12] H. J. Christen, F. Hanefeld, H. Eiffert, and R. Thomssen, "Epidemiology and clinical manifestations of Lyme borreliosis in childhood. A prospective multicentre study with special regard to neuroborreliosis," Acta Paediatrica, vol. 386, pp. 1-75, 1993.

[13] M. Peltomaa, H. Saxen, I. Seppälä, M. Viljanen, and I. Pyykkö, "Paediatric facial paralysis caused by Lyme borreliosis: a prospective and retrospective analysis," Scandinavian Journal of Infectious Diseases, vol. 30, no. 3, pp. 269-275, 1998.

[14] K. E. Campbell and J. F. Brundage, "Effects of climate, latitude, and season on the incidence of Bell's palsy in the US Armed Forces, October 1997 to September 1999," The American Journal of Epidemiology, vol. 156, no. 1, pp. 32-39, 2002.

[15] E. Peitersen, "Bell's palsy: the spontaneous course of 2,500 peripheral facial nerve palsies of different etiologies," Acta OtoLaryngologica, no. 549, pp. 4-30, 2002.

[16] A. Biebl, E. Lechner, K. Hroncek et al., "Facial nerve paralysis in children: is it as benign as supposed?" Pediatric Neurology, vol. 49, no. 3, pp. 178-181, 2013.

[17] K. K. Adour, F. M. Byl, R. L. Hilsinger Jr., Z. M. Kahn, and M. I. Sheldon, "The true nature of Bell's palsy: analysis of 1,000 consecutive patients," Laryngoscope, vol. 88, no. 5, pp. 787-801, 1978.

[18] T. S. Shafshak, "The treatment of facial palsy from the point of view of physical and rehabilitation medicine," Europa Medicophysica, vol. 42, no. 1, pp. 41-47, 2006.

[19] G. M. Thaera, K. E. Wellik, D. M. Barrs, E. D. D. Dunckley, D. M. Wingerchuk, and B. M. Demaerschalk, "Are corticosteroid and antiviral treatments effective for bell palsy? A critically appraised topic," Neurologist, vol. 16, no. 2, pp. 138-140, 2010.

[20] E. Unüvar, F. Oğuz, M. Sidal, and A. Kiliç, "Corticosteroid treatment of childhood Bell's palsy," Pediatric Neurology, vol. 21, no. 5, pp. 814-816, 1999. 
[21] L. J. Teixeira, B. G. Soares, V. P. Vieira, and G. F. Prado, "Physical therapy for Bell's palsy (idiopathic facial paralysis)," Cochrane Database of Systematic Reviews, no. 16, Article ID CD006283, 2008. 


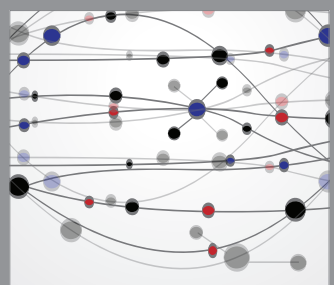

The Scientific World Journal
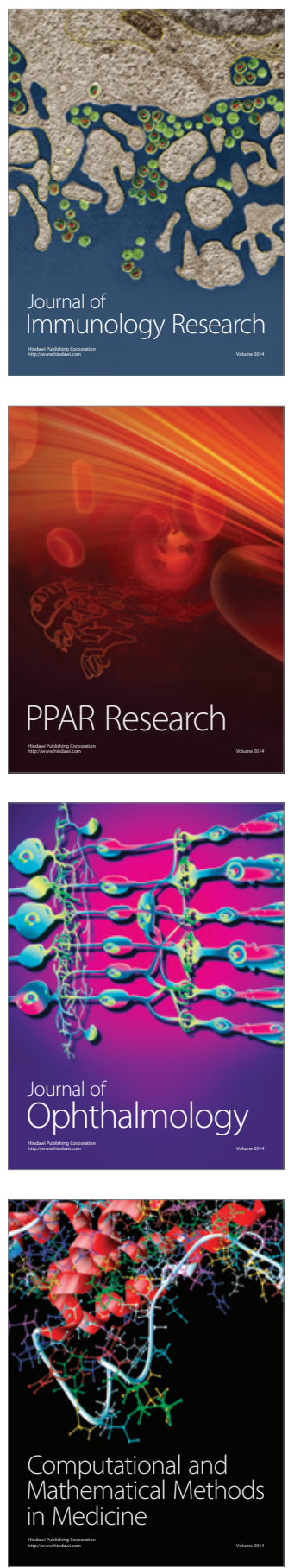

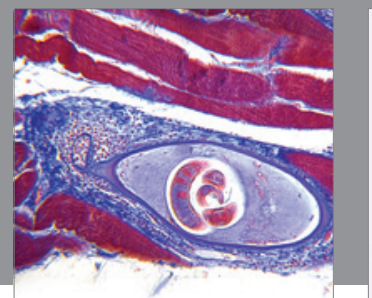

Gastroenterology

Research and Practice
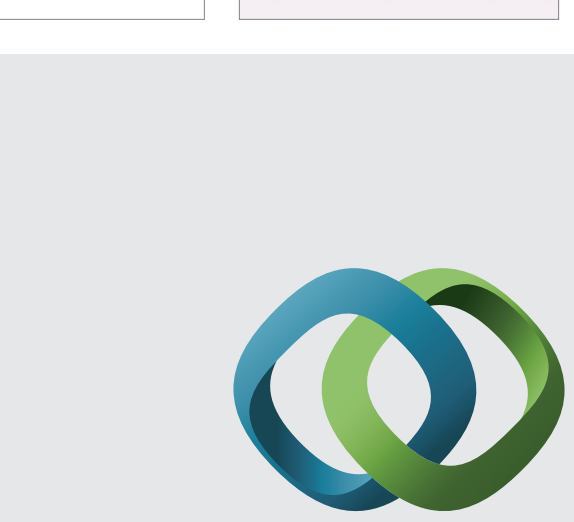

\section{Hindawi}

Submit your manuscripts at

http://www.hindawi.com
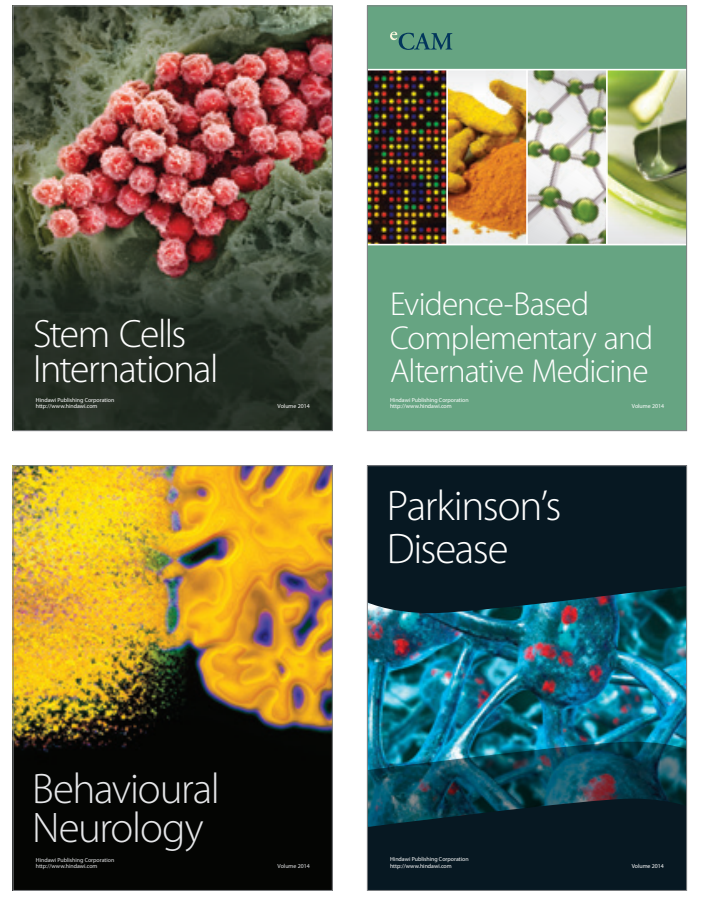
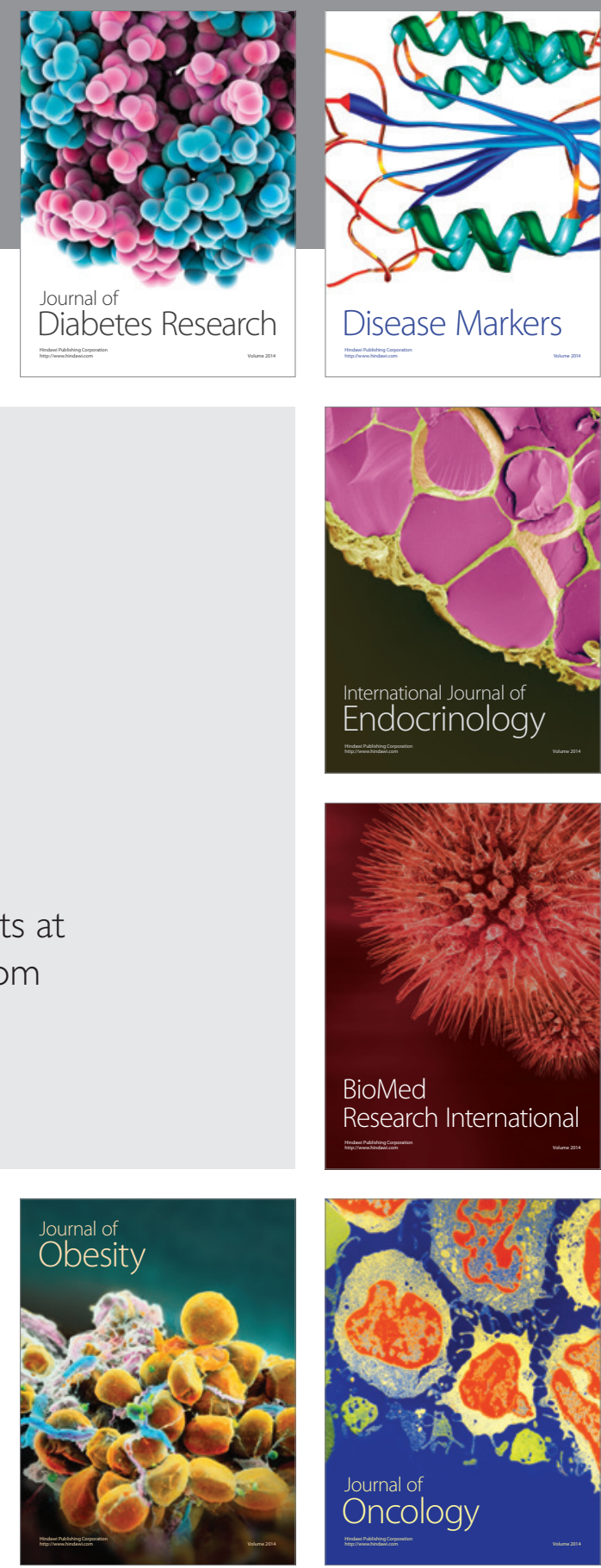

Disease Markers
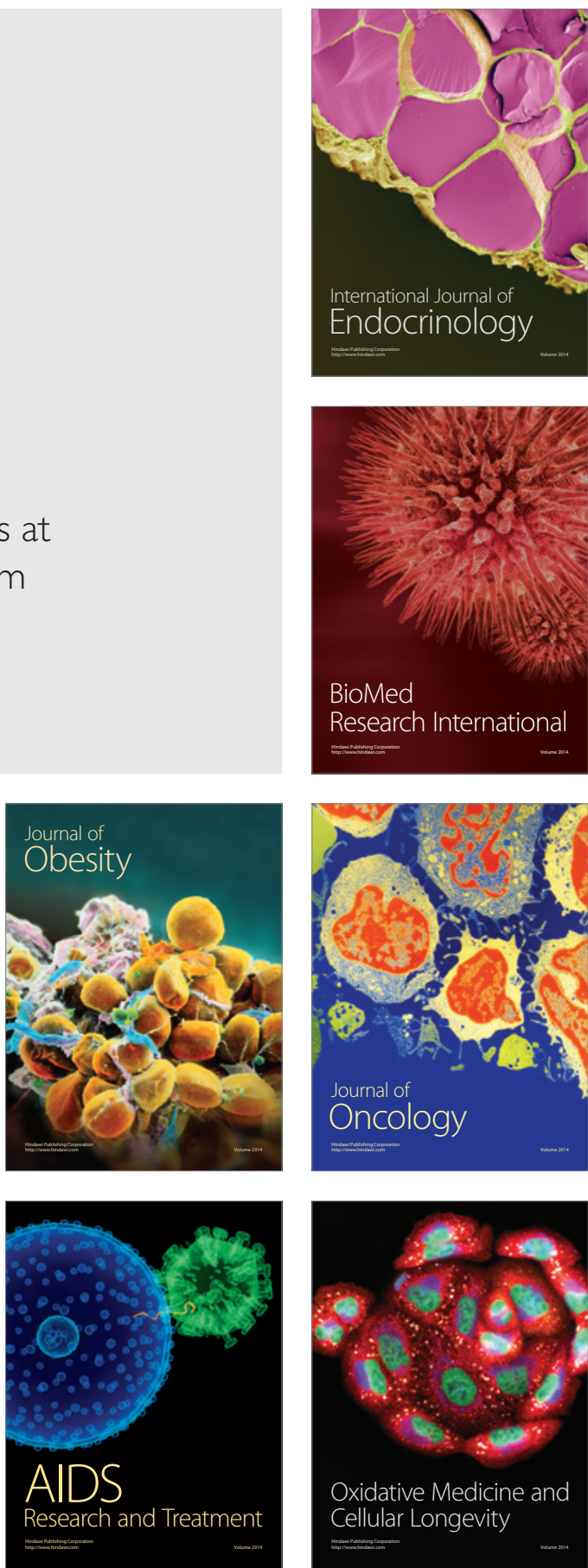